\title{
Acute exacerbation of idiopathic pulmonary fibrosis: report of a series
}

\author{
V. Ambrosini*, A. Cancellieri" ${ }^{\#}$ M. Chilosi ${ }^{\top}$, M. Zompatori ${ }^{+}$, R. Trisolini ${ }^{\S}$, L. Saragonif ${ }^{f}$, V. Poletti*
}

Acute exacerbation of idiopathic pulmonary fibrosis: report of a series. V. Ambrosini, A. Cancellieri, M. Chilosi, M. Zompatori, R. Trisolini, L. Saragoni, V. Poletti. (C) ERS Journals Ltd 2003.

ABSTRACT: This study describes five cases presenting an acute clinical course of pulmonary fibrosis, in the absence of specific precipitating factors.

A retrospective chart review of five patients with histologically proved usual interstitial pneumonia was carried out in 2001-2002. Clinical data, bronchoalveolar lavage (BAL) findings, high resolution computed tomography and histological features were reported.

On admission all cases presented hypoxemia and dyspnoea, while some showed an increase of carbohydrate antigen 19.9 or laboratory tests typical of infection, although appropriate cultures were all negative. Altogether, four subjects died and only one is on follow-up. A pattern of diffuse ground-glass or alveolar opacification superimposed on reticular and linear findings was evident on lung imaging in all cases. Marked neutrophilia, together with type II reactive cells hyperplasia, was detected on BAL.

Histological findings, from open lung biopsy or autopsy, showed all the aspects of usual interstitial pnemonia with superimposed features of acute lung injury, such as diffuse alveolar damage, with or without hyaline membranes, type II reactive cells hyperplasia and numerous fibroblastic foci. This study also underlines the diagnostic value of bronchoalveolar lavage versus open lung biopsy.

Eur Respir J 2003; 22: 821-826.
*Dept of Thoracic Diseases, and ${ }^{f}$ Dept of Anatomic Pathology, Ospedale Giovanni Battista Morgagni, Forlì, ${ }^{\#}$ Dept of Anatomic Pathology, and $\$$ Thoracic Endoscopic Unit, Ospedale Maggiore, Bologna, "Dept of Pathology, University of Verona, Verona, and ${ }^{+}$Institute of Radiology, University of Parma, Parma, Italy.

Correspondence: V. Poletti, Dipartimento di Malattie dell'Apparato Respiratorio e del Torace, U.O. Endoscopia Toracica, Ospedale G.B. Morgagni, Piazzale Solieri 1, 47100 Forlì. Fax: 390543731461

E-mail: vepolet@tin.it

Keywords: Acute respiratory failure, bronchoalveolar lavage, diffuse alveolar damage, highresolution computed tomography, idiopathic pulmonary fibrosis

Received: March 12003

Accepted after revision: June 122003
Idiopathic pulmonary fibrosis (IPF) is a chronic lung disease of unknown cause, limited to the lungs, associated with a histological pattern of usual interstitial pneumonia (UIP) [1] on lung biopsy. Onset is usually gradual, with dyspnoea the most prominent symptom.

Basal and peripheral reticular opacities, associated with traction bronchiectasis and honeycombing, are typical highresolution computed tomography (HRCT) signs of IPF in most cases [1,2] and these findings, combined with the clinical profile, are sufficient to establish a confident diagnosis of IPF, even in the absence of a surgical lung biopsy [2].

The natural history is invariably one of gradual and progressive deterioration, with median length of survival from the time of diagnosis ranging 2.5-3.5 yrs. Although chronic in nature, one complication that has received little attention in the literature [3-5] is an accelerated phase of the disease, leading to death in a period of a few weeks to a few months [4]. It still remains uncertain what causes such accelerated clinical deterioration. The histological findings from lung biopsy specimens show variable aspects; the typical UIP pattern is associated with signs of acute lesions, such as diffuse alveolar damage (DAD) with or without hyaline membranes, numerous fibroblastic foci, organising pneumonia and haemorrhage with capillaritis [6]. Bronchoalveolar lavage (BAL) shows a marked neutrophilia [3, 5].

The incidence of this clinical condition is not well known and its clinical profile, morphological pattern, laboratory data, HRCT findings and treatment are not yet well defined [7]. Therefore, a series of five cases of accelerated IPF, with histopathologically proved UIP associated with superimposed acute lesions, is presented in detail in the current study.

\section{Subjects and methods}

The study group consisted of five subjects (four males, one female) with IPF diagnosed on the basis of American Thoracic Society (ATS)/European Respiratory Society criteria [1] and/or confirmed by a UIP pattern on surgical biopsy or autopsy. Data were collected from May 2001 to June 2002.

None of the patients had a history for other known causes of interstitial lung disease, such as drug toxicity, environmental exposures or collagen vascular disease. All patients were admitted to hospital for worsening of dyspnoea, except for case no. 3, who entered the emergency ward for an accidental fall without loss of consciousness.

After admission, acute respiratory failure developed in all cases in the absence of any precipitating factor. Accelerated phase of IPF was defined as follows (modified from AKIRA et al. [4]): exacerbation of dyspnoea within one month, newly developing diffuse pulmonary opacities on chest radiographs, arterial oxygen tension $\left(\mathrm{Pa}, \mathrm{O}_{2}\right) /$ inspiratory oxygen fraction $\left(\mathrm{Fi}_{1}, \mathrm{O}_{2}\right)$ $<30 \mathrm{kPa}$, absence of heart congestive failure or infection.

Collected data included tobacco smoke exposure, symptoms and clinical evaluation on admission, laboratory findings, results of microbial cultures, treatment before admission, need of mechanical ventilation, survival days in hospital and follow-up. An analysis of blood gases was carried out on all subjects.

Pulmonary function tests (PFT), which included spirometry (predicted normal values were obtained from standard references and the results expressed as \% predicted) and carbon monoxide diffusing capacity, were performed by two patients. 
Table 1.-Clinical data on admission

\begin{tabular}{|c|c|c|c|c|c|}
\hline \multirow[t]{2}{*}{ Variable } & \multicolumn{5}{|c|}{ Case no. } \\
\hline & 1 & 2 & 3 & 4 & 5 \\
\hline Sex & M & M & $\mathrm{F}$ & M & M \\
\hline Age yrs & 72 & 65 & 80 & 67 & 67 \\
\hline Smoker & NA & Exsmoker & NA & Exsmoker & Exsmoker \\
\hline Dyspnoea & + & + & + & + & + \\
\hline Dry cough & - & - & + & - & + \\
\hline Clubbing & + & - & - & - & + \\
\hline Inspiratory crackles & + & + & + & + & + \\
\hline Hypoxemia & + & + & + & + & + \\
\hline Fever & - & + & - & + & + \\
\hline Corticosteroid therapy & + & + & - & + & + \\
\hline Duration & 6 days & 2 months & & 2 months & $3 \mathrm{yrs}$ \\
\hline Immunosuppressive therapy & - & - & - & $\mathrm{Az}$ & Cy \\
\hline Duration & & & & 7 days & 3 yrs \\
\hline $\mathrm{O}_{2}$ therapy & + & + & - & + & - \\
\hline $\mathrm{Pa}, \mathrm{O}_{2} / F_{1}, \mathrm{O}_{2} \mathrm{kPa}$ & 9.44 & 29.66 & 11.97 & 29.39 & 29.79 \\
\hline
\end{tabular}

M: male; F: female; NA: not applicable; +: yes; -: no; Az: azathyoprine; Cy: cyclophosfamide; $\mathrm{O}_{2}$ : oxygen; $P$ a, $\mathrm{O}_{2}$ : arterial oxygen tension; $F \mathrm{i}, \mathrm{O}_{2}$ : inspiratory oxygen fraction.

All subjects were submitted to an HRCT scan of the chest. For this diagnostic investigation, $1 \mathrm{~mm}$ thick images obtained at $10 \mathrm{~mm}$ intervals through the chest were taken. Scans were reconstructed using a high spatial-frequency algorithm.

Fibreoptic bronchoscopy (FBS) was performed on four subjects. One patient (no. 2) could not be submitted to FBS due to critical clinical conditions. The BAL was performed by instillation of six $25 \mathrm{~mL}$ aliquots of sterile saline solution warmed at $37^{\circ} \mathrm{C}$; fluid was aspirated immediately after each aliquot was instilled and collected in a sterile container. After recovery, BAL fluid was filtered through a monolayer of surgical gauze to remove mucus, and immediately centrifugated at $34 \times g$ for $7 \mathrm{~min}$. Cytospin preparations were stained by both the Diff Quick and the Papanicolau methods. A BAL specialist (V. Poletti) performed the differential cell count (under microscopy at $\times 1000$ by counting 300 cells in random fields) and the evaluation of cytological cells characteristics. Total count was obtained using a haemocytometer (Sysmex SE9500; Dasit, Milan, Italy).

On BAL fluid and sputum samples, routine cultures for bacteria, mycobacteria and fungi, cell vial cultures and immunofluorescent tests for cytomegalovirus (CMV), Herpes simplex virus type I, Adenoviruses, influenza and parainfluenza viruses, respiratory syncitial virus (RSV) and Legionella were performed. Serological tests for RSV, Adenovirus, CMV, HSV, and Legionella, were also carried out. Urinary antigen test for the Legionella pneumophila serogroup and detection of the $65 \mathrm{kDa}$ CMV-related lower matrixphosphoprotein (pp65) in the nuclei of the peripheral blood polymorphonuclear leukocytes completed the microbiological investigations. All samples for microbiological analysis were taken before any antibiotic therapy was instituted.

None of the subjects were at risk of adult respiratory distress syndrome, nor had evidence of left ventricular dysfunction.

Three subjects (cases no. 1, 3 and 4) underwent both transbronchial and open lung biopsy (OLB). Autopsy was carried out in all patients who died, except for case no. 4. Formalin-fixed, paraffin-embedded large tissue samples were available for all cases. For each subject, two to six haematoxylin/eosin stained slides were reviewed. The presence and the extent of hyaline membranes, vascular thrombi, organisation, type II pneumocyte hyperplasia in DAD areas, fibroblastic foci, honeycombing in UIP areas and the DAD:UIP were evaluated. Pathological abnormalities were assessed using a $0-3$ scale $(0=$ absence of the lesion, $1=$ the lesion is present in $<30 \%$ of the examined material, $2=30-60 \%, 3=>60 \%$ ). Intracytoplasmatic fibrin was observed in the only case in which clear-cut hyaline membranes were missing (case no. 4). Pathological samples (OLB and autopsy specimens) were investigated using monoclonal antibodies against herpes virus type 1, CMV and with specials stains for Pneumocystis carinii, fungi (Grocott stain) and acid fast bacilli (Ziehl Neelsen stain).

\section{Results}

Clinical and laboratory data on admission are shown in table 1 and table 2 . The mean age was 70 yrs (range 65-80). The time between the diagnosis of IPF and the onset of the accelerated phase ranged from 3 months to $3 \mathrm{yrs}$.

All subjects presented dyspnoea and hypoxemia, three had fever, whereas only two (cases no. 3 and 5) had dry cough. Inspiratory crackles were heard on physical examination in all cases and in two (cases no. 1 and 5) digital clubbing was present.

Four subjects (cases no. 1, 2, 4, and 5) were receiving corticosteroid treatment at the time of hospital admission (case no. 1 methylprednisolone $60 \mathrm{mg} \cdot \mathrm{day}^{-1}$, no. 2 prednisone

Table 2. - Laboratory data on admission

\begin{tabular}{lccccccc}
\hline Variable & & \multirow{2}{*}{$\mathrm{NR}$} & \multicolumn{5}{c}{ Case no. } \\
\cline { 4 - 8 } & & & 1 & 2 & 3 & 4 & 5 \\
\hline WBC & $10^{3} \cdot \mathrm{mm}^{-3}$ & $4.0-10.0$ & 13.0 & 7.90 & 9.12 & 11.30 & 10.63 \\
& $\mathrm{~N} \%$ & $45-74$ & 80.5 & 60.5 & 61.8 & 76.1 & 78.0 \\
& $\mathrm{~L} \%$ & $16-45$ & 8.3 & 28.0 & 25.2 & 20.3 & 11.4 \\
& $\mathrm{E} \%$ & $0-7$ & 0.0 & 2.4 & 3.8 & 0.9 & 0.8 \\
PTL & $10^{3} \cdot \mathrm{mm}^{-3}$ & $130-400$ & 255 & 41 & 194 & 259 & 227 \\
Fibrinogen & $\mathrm{mg} \cdot \mathrm{dL}^{-1}$ & $200-400$ & 887 & $\mathrm{NA}$ & 349 & 399 & 668 \\
ESR & $\mathrm{mm} \cdot \mathrm{h}^{-1}$ & $<15$ & $\mathrm{NA}$ & 43 & 19 & 17 & 60 \\
CRP & $\mathrm{mg} \cdot \mathrm{dL}^{-1}$ & $1-2$ & 16.4 & 1.0 & $\mathrm{NA}$ & 1.1 & 14.1 \\
Creatinine & $\mathrm{mg} \cdot \mathrm{dL}^{-1}$ & $<1.5$ & 0.77 & 0.74 & 0.73 & 0.78 & 1.52 \\
LDH & $\mathrm{U} \cdot \mathrm{dL}^{-1}$ & $100-190$ & $\mathrm{NA}$ & 625 & $\mathrm{NA}$ & 857 & 761
\end{tabular}

NR: normal ranges; WBC: white blood cells; N: neutrophils; L: lymphocytes; E: eosinophils; PTL: platelets; NA: not applicable; ESR: erythrocyte sedimentation rate; CRP: C-reactive protein; LDH: lactate dehydrogenase. 
$50 \mathrm{mg} \cdot \mathrm{day}^{-1}$, no. 4 prednisone $75 \mathrm{mg} \cdot \mathrm{day}^{-1}$ and no. 5 prednisone $25 \mathrm{mg} \cdot \mathrm{day}^{-1}$ ). Three patients (cases no. 1, 2 and 4) were on oxygen $\left(\mathrm{O}_{2}\right)$ therapy. Cases no. 4 and 5 underwent immunosuppressive therapy with azathioprine and cyclophosfamide respectively. Three subjects (cases no. 2, 4 and 5) were exsmokers.

Although only three subjects (cases no. 1, 4 and 5) presented an increase of white blood cells (WBC) on admission, elevated values of either erythrocyte sedimentation rate (ESR) or reactive $\mathrm{C}$ protein $(\mathrm{RCP})$ were found in all patients. Increased fibrinogen levels were determined on admission in four cases.

Thrombocytopenia was present on admission in one case (no. 2). Lactate dehydrogenase (LDH) values were increased in three subjects (cases no. 2, 4 and 5).

At the time of the diagnosis of IPF, a restrictive impairment was found in all but one case (in case no. 3 PFT were not feasible) and was documented by reduced total lung capacity (TLC). TLC $\%$ of predicted value (normal range 47-76\%) was $76 \%, 62 \%, 47 \%$, and $73 \%$ in cases no. 1-5, respectively). Room air hypoxemia was present in all subjects $\left(\mathrm{Pa}, \mathrm{O}_{2} / \mathrm{Fi}, \mathrm{O}_{2}\right.$ was $35.5,36.7,45.0,38.6$ and $41.1 \mathrm{kPa}$ in cases no. $1-5$, respectively). The only two subjects who were able to perform PFT after admission for worsening of dyspnoea showed a restrictive pattern of ventilatory defect (TLC $\%$ pred was $60 \%$ in case no. 2 and $47 \%$ in no. 4). Carbon monoxide diffusing capacity was severely decreased in case no. 2 , whereas in case no. 4 the test was not feasible. At the time of acute exacerbation all subjects presented a $\mathrm{Pa}, \mathrm{O}_{2} / F_{1}, \mathrm{O}_{2}$ ratio $<30 \mathrm{kPa}$ (table 1 ).

\section{Laboratory findings}

Although at the time of accelerated deterioration, four subjects presented an increase of WBC, case no. 2 showed a decrease of both WBC and platelets. Cases no. 2, 4 and 5 showed further increase of LDH levels after the onset of the accelerated phase. Infection as a possible cause for rapid deterioration was ruled out, taking into account the fact that appropriate cultures on blood, urine, sputum, immunofluorescent direct tests, pp65 protein in the peripheral blood leukocytes and immunohistochemical evaluations on biopsy or autopsy specimens, were all negative. Only case no. 5 developed positive sputum cultures for Hemophilus parainfluenzae and Acinetobacter iwoffii a few days before death. Elevated values of carbohydrate antigen 19.9 were present in case no. $2\left(55 \mathrm{U} \cdot \mathrm{L}^{-1}\right)$ and no. $5\left(466 \mathrm{U} \cdot \mathrm{L}^{-1}\right)$.

\section{Thoracic imaging}

In all cases typical signs of IPF were found (reticular pattern more prominent at the lungs bases, traction bronchiectasis and areas of honeycombing).

Accelerated clinical deterioration was associated with progressive bilateral shadowing of lung parenchyma in all cases, on either chest radiography or HRCT. Areas of groundglass attenuation were diffuse (fig. 1) in cases no. 4 and no. 5 ,

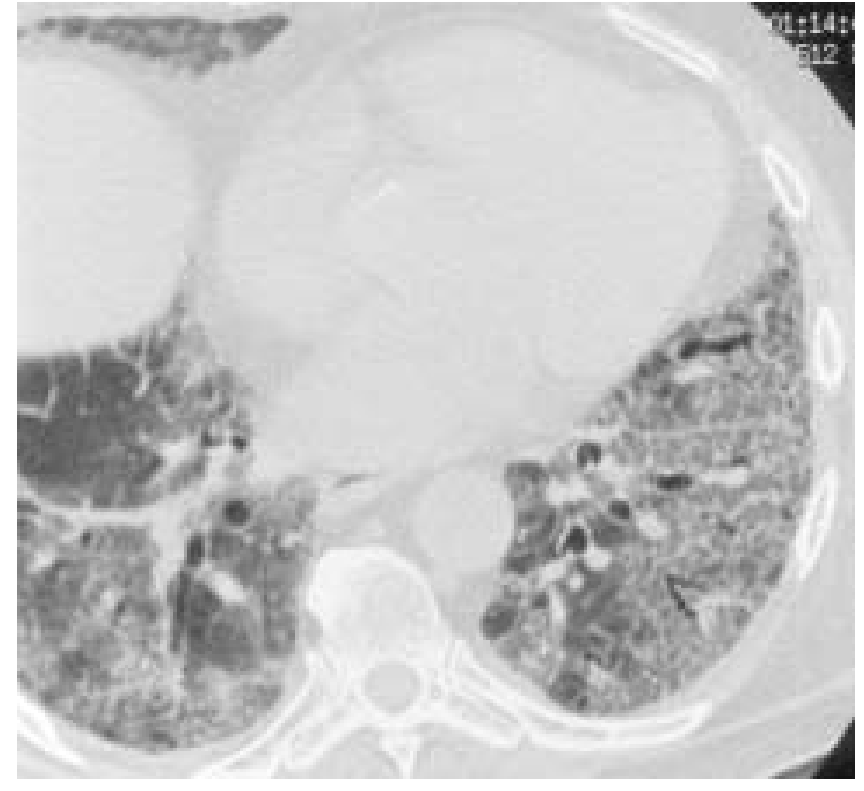

Fig. 1. - High-resolution computed tomography scan at the lung bases level: reticular and linear pattern with associated bronchioloectases and diffuse ground glass attenuation.

patchy in case no. 1, and areas of alveolar diffuse opacification were diffuse in case no. 2 and no. 3. Such abnormalities resolved after the acute phase in the only survivor (case no. 1).

\section{Bronchoalveolar lavage profile}

The cytological profile showed a slight increase in total cell number and a marked neutrophilia in all cases (table 3 ). Type II reactive cells were present in all cases. Lymphocyte subset analysis showed an increased CD4/CD8 ratio in three cases and a decreased ratio in one case. Pulmonary pathogens did not grow in BAL cultures and neoplastic cells were not found.

\section{Mechanical ventilation}

When peripheral blood $\mathrm{O}_{2}$ saturation dropped $<72 \%$, case no. 1 underwent noninvasive mechanical ventilation for 24 days. Invasive mechanical ventilation was required for case no. 3 (peripheral blood $\mathrm{O}_{2}$ saturation $78 \%$ before intubation) for 28 days. In the other cases only comfort and palliative measures were carried out.

\section{Biopsy/autopsy}

Pathological score is summarised in table 4. Various combinations of typical UIP and DAD areas were present

Table 3. - Brochoalveolar lavage profile

\begin{tabular}{|c|c|c|c|c|c|c|}
\hline Case no. & Cellularity $10^{3} \cdot \mathrm{mm}^{-3}$ & Macrophages \% & Lymphocytes \% & Neutrophils \% & Eosinophils \% & CD4:CD8 \\
\hline & $(<230)$ & $(>90)$ & $(<15)$ & $(<4)$ & $(<0.9)$ & $(1-2.4)$ \\
\hline 1 & 240 & - & 12 & 35 & 1 & 4.12 \\
\hline 2 & NA & NA & NA & NA & NA & NA \\
\hline 3 & - & 7 & 1 & 89 & 3 & 2.53 \\
\hline 4 & 250 & 5 & 3 & 90 & - & 7.00 \\
\hline 5 & 80 & 87 & 2 & 11 & - & 0.10 \\
\hline
\end{tabular}

Data are presented as $\mathrm{n}$ with normal ranges in brackets. NA: not applicable. 
Table 4.-Semiquantitative analysis of histopathological findings

\begin{tabular}{lccccc}
\hline & \multicolumn{5}{c}{ Case no. } \\
\cline { 2 - 6 } & 1 & 2 & 3 & 4 & 5 \\
\hline Honeycombing & 1 & 2 & 3 & 2 & 1 \\
Fibroblastic foci & 1 & $1: 2$ & $1: 2$ & 1 & $1: 2$ \\
Squamous metaplasma & 1 & 1 & 3 & 2 & 1 \\
Vascular thrombosis & 0 & 1 & 1 & 0 & 0 \\
Hyaline membranes/fibrin & 1 & 2 & 2 & $0: 1$ & 2 \\
Type II cells hyperplasia & 1 & 2 & 2 & 1 & 1 \\
Organising stage & 3 & 0 & 0 & 3 & 0 \\
DAD/UIP areas & $1: 1.5$ & $3: 1$ & $2: 1$ & $1: 2.5$ & $2: 1$ \\
\hline
\end{tabular}

Each histological feature was scored as follows: $0=a b s e n t ; 1=$ present in $<30 \%$ of examined material; $2=30-60 \%$ of examined material; $3=>60 \%$. DAD: diffuse alveolar damage; UIP: usual interstitial pneumonia.

in all cases, with a DAD/UIP ratio ranging 3:1-1:1.5. All samples showed type II pneumocyte hyperplasia and fibroblastic foci, although the latter were very rare in cases no. 2, 3 and 5. Squamous metaplasia and honeycombing were found in all samples, particularly in case no. 3, in which they were extensive. Hyaline membranes were observed in all cases but one (case no. 4), in which, however, intracytoplasmatic fibrin was present in DAD areas. Organisation of the fibrinous exudates was either absent (cases no. 2, 3 and 5) or prominent (cases no. 1 and 4) (fig. 2). Prominent organisation associated with the lowest DAD/UIP ratio was found in cases no. 1 and 4 , one of which refers to the only surviving patient.

\section{Therapy and outcome}

All patients were treated with high doses of methylprednisolone $\left(500-1000 \mathrm{mg} \cdot \mathrm{day}^{-1}\right.$ for 3 days and then $\left.125 \mathrm{mg} \cdot \mathrm{day}^{-1}\right)$ and cyclophosfamide was administered in a bolus of $500 \mathrm{mg} \cdot \mathrm{m}^{-2}$ every 3 weeks. $\mathrm{O}_{2}$ therapy and antibiotic therapy (case no. 1: ceftriaxone and moxifloxacin; case no. 2: ceftriaxone; case no. 3: ceftriaxone and gentamicin, followed by ceftazidime and klaritromicin; case no. 4: imipemen and cilastatin followed by nizatidine and ceftazidime; case no. 5: imipemen) were instituted in all cases.

In total, four subjects died. The time from rapid deterioration through to death averaged 13 days (range 7-30) (table 5). Case no. 1 was the only survivor. The natural course of case no. 1 was characterised by the development of an acute episode of loss of consciousness for $20 \mathrm{~min}$, associated with marked hypoxemia. The neurological physical exam showed left harm hemiplegia. A CT head scan detected an ischemic stroke. Pulmonary function progressively improved and cyclophosfamide $\left(100 \mathrm{mg} \cdot \mathrm{day}^{-1}\right)$ was introduced before being released from the hospital. At 1.5 yrs from hospital discharge,

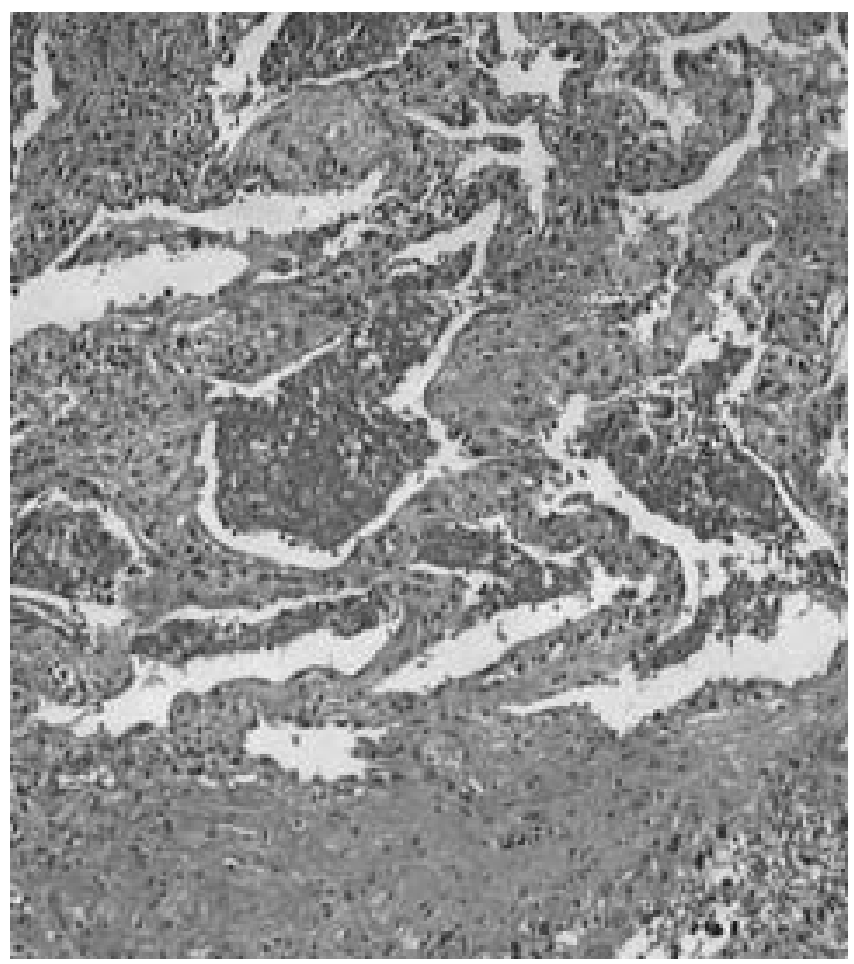

Fig. 2.-Open lung biopsy: intra-alveolar septal proliferation of myofibroblasts embedded in a pale extracellular matrix. Type II cells hyperplasia is also evident (haematoxilyn and eosin stain, low power magnification).

the patient is clinically stable, undergoing only $\mathrm{O}_{2}$ and corticosteroid therapy.

\section{Discussion}

Evidence of a different acute clinical course of IPF has been recently reported by various authors [1, 3-5, 8, 9]. Exacerbation of respiratory failure develops in some cases as a consequence of the occurrence of precipitating factors [10], such as pulmonary or nonpulmonary infections $(6.5 \%)$, pulmonary embolism $(3.4 \%)$, heart failure $(14.4 \%)$, bronchogenic carcinoma $(10.4 \%)$, ischemic heart disease $(9.5 \%)$ and stroke. However, the clinical course of a subset of IPF patients is characterised by the onset of an accelerated phase of the illness [4], in the absence of any identifiable precipitating cause, clinically resembling the acute respiratory distress syndrome.

The main clinical/pathological characteristics, lung imaging, laboratory and BAL fluid findings of a series of five patients with IPF developing an accelerated phase is reported

Table 5. - Clinical outcome

\begin{tabular}{|c|c|c|c|c|c|}
\hline \multirow[t]{2}{*}{ Variable } & \multicolumn{5}{|c|}{ Case no. } \\
\hline & 1 & 2 & 3 & 4 & 5 \\
\hline Illness duration ${ }^{\#}$ & 3 months & 3 months & $1 \mathrm{yr}$ & 3 months & 3 yrs \\
\hline $\begin{array}{l}\text { Mechanical ventilation } \\
\text { Duration }\end{array}$ & $\begin{array}{c}\text { Yes } \\
24 \text { days }\end{array}$ & No & $\begin{array}{c}\text { Yes } \\
28 \text { days }\end{array}$ & No & No \\
\hline Biopsy (OLB or TLB) & Both & No & Both & Both & No \\
\hline Autopsy & - & + & + & - & + \\
\hline Outcome & Alive and well & Died at day 7 & Died at day 30 & Died at day 9 & Died at day 7 \\
\hline
\end{tabular}

OLB: open lung biopsy; TLB: transbronchial lung biopsy. ${ }^{*}$ : time between diagnosis of idiopathic pulmonary fibrosis and onset of accelerated phase. 
in detail. The diagnosis of IPF was based on clinical and/or radiographical ATS criteria and confirmed by the findings of a UIP pattern on biopsy or autopsy. At the time of acute exacerbation all subjects met Akira's criteria for acute exacerbation of IPF.

A male preponderance of subjects was evident. All subjects presented hypoxemia and dyspnoea. End-inspiratory crackles were heard in all patients, while two had dry cough. Three subjects had fever. Acute respiratory failure developed in the absence of any signs of infection (serological tests and cultures from blood, urine, BAL were all negative) or other precipitating cause. Only one subject survived.

From the evaluation of this series, there are no definite factors that can contribute to the estimate of when an IPF patient will enter the acute phase, as shown by the wide time range of illness duration before the onset of the accelerated phase. Other authors reported a short time between the diagnosis of IPF and the acute exacerbation with a UIP and DAD pattern on biopsy (ranging 1-65 months) [11].

Laboratory findings suggested the presence of an infection (elevated neutrophils, ESR, RCP and fibrinogen in some cases, thrombocytopenia in one), but such hypothesis as a possible cause of respiratory failure was ruled out as appropriate cultures, immunofluorescent and immunhistochemical tests were all negative. One case developed positive sputum cultures for $H$. parainfluenzae and $A$. iwoffii a few days before death, whereas all previous samples were negative, suggesting that such an infectious agent could not be responsible for the onset of the acute phase. It should be noted that PCR was not performed on BAL or bioptic samples. However, at present this technique is not routinely used in the diagnosis of respiratory infection in patients affected by chronic lung disease [4, 7]. Furthermore, although it seems to be more sensitive, its clinical value in non-human immunodeficiency virus immunocompromised hosts has not yet been determined $[12,13]$. Marked increased levels of LDH also suggest the hypothesis of acute lung injury (as in acute respiratory distress syndrome or respiratory failure associated with acute interstitial pneumonia (AIP)) at the basis of the acute clinical deterioration.

Clinical, pathological and chest imaging findings from IPF patients during the accelerated phase are very similar to those of subjects developing AIP [14]. This is a fulminant form of lung injury that develops in previously healthy individuals [1]. Patients presenting a focal lung involvement improve, whereas those with a massive lung injury die, with an overall survival rate of $\sim 50 \%$. Since all cases presented in the current study had a classical bibasilar subpleural reticular and linear pattern in HRCT scans taken before the onset of the acute exacerbation, they cannot be described as AIP [14].

Histopathological findings of specimens obtained during the accelerated phase of IPF show the presence of a UIP pattern associated with signs of DAD, such as hyaline membranes and type II reactive cells hyperplasia. Various authors described similar findings in cases of accelerated IPF [4, 9], whereas KONDOH et al. [3] reported a series of three in which hyaline membranes were absent and other features of acute lesions were not described. However, in the current study, in the only case in which hyaline membranes were not clearly detected, the acute lung injury pattern was characterised by interstitial and intra-alveolar organisation and type II cells intracytoplasmatic fibrin. Among the cases with the lowest DAD:UIP (cases no. 1 and 4), case no. 1 is the only survivor of the series, suggesting that lower extension of DAD areas is associated with a better prognosis. The histopathological differential diagnosis for UIP pattern includes mainly the fibrosing NSIP pattern and the DIP pattern. DAD in the organisation stage may be confused with UIP pattern in small specimens but in OLB and autopsy samples the distribution of the lesions are quite different, as fibrosing DAD is homogeneously distributed, while UIP is mainly located in subpleural region. In the current study the hetereogenous and subpleural distribution of the lesion was always evident.

AKIRA et al. [4] has described the HRCT features of accelerated IPF. In this study $50 \%$ of subjects with a multifocal disease and almost $100 \%$ of those with diffuse abnormalities died and presented a pattern of DAD superimposed on UIP on biopsy. The peripheral distribution was associated with the presence of numerous fibroblastic foci on biopsy and with a better response to treatment. The only survivor in the current series had areas of ground glass attenuation with a patchy distribution superimposed on the classical IPF pattern.

BAL was helpful in both ruling out other possible causes of respiratory failure, such as infection and alveolar haemorrhage, and achieving a correct diagnosis. In fact, together with an increased cellularity and a marked increase of the neutrophil percentage, the presence of type II reactive cells suggest that pathological findings cannot be those of UIP alone, but are also associated with signs of acute lung injury.

Considering the high mortality rate of such critically ill patients [15] and the possibility of other less invasive procedures useful for diagnosis, various authors agree that OLB should be performed on selected subjects with an uncertain diagnosis or when they are thought not to have IPF [2]. From an evaluation of the series reported here, it seems that the presence of reactive cells in BAL specimens of a patient with rapidly deteriorating IPF might be sufficient for a correct diagnosis of accelerated IPF.

The current study confirms that idiopathic pulmonary fibrosis patients may develop an accelerated phase of the disease in the absence of any identifiable precipitating cause, associated with pathological findings of usual interstitial pneumonia and variable aspects of diffuse alveolar damage, with or without hyaline membranes. The diagnostic value of fibreoptic bronchoscopy/bronchoalveolar lavage and lung imaging is also underlined. The need of open-lung biopsy is therefore to be questioned and further studies on larger groups are needed to better evaluate these aspects.

\section{References}

1. American Thoracic Society/European Respiratory Society international multidisciplinary consensus classification of the idiopathic interstitial pneumonias. Am J Respir Crit Care Med 2002; 165: 277-304.

2. Hunninghake GW, Zimmerman MB, Schwartz DA, et al. Utility of lung biopsy in the diagnosis of idiopathic pulmonary fibrosis. Am J Respir Crit Care Med 2001; 164: 193-196.

3. Kondoh $\mathrm{Y}$, Taniguchi $\mathrm{H}$, Kawabata $\mathrm{Y}$, Toyoharu $\mathrm{Y}$, Suzuki K, Takagi K. Acute exacerbation in idiopathic pulmonary fibrosis: analysis of clinical and pathological findings in three cases. Chest 1993; 103: 1808-1812.

4. Akira M, Hamada H, Sakatani M, Kobayashi C, Nishioka M, Yamamoto S. CT findings during accelerated deterioration in patients with idiopathic pulmonary fibrosis. $A m J$ Roentgenol 1997; 168: 79-83.

5. Bona R, Macchiarulo R, Trabalzini P, Vacca A, Marchisio U, Mosso L. Evoluzione critica in un caso di fibrosi polmonare idiopatica [Critical outcome of a case of idiopathic fibrosis]. Minerva Anestesiol 1995; 61: 425-429.

6. Travis WD, Colby TV, Koss MN, Rosado-de-Christenson ML, Muller NL, King TE. Non neoplastic disorders of the lower respiratory tract. In: West King D, ed. Atlas of non-tumor pathology. Washington DC, American Registry of Pathology and Armed Forces Institute of Pathology, 2002; pp. 6869. 
7. Saydin G, Islam A, Afessa B, Ryu H, Scott JP, Peters S Outcome of patients with idiopathic pulmonary fibrosis admitted to the intensive care unit. Am J Respir Crit Care Med 2002; 166: 839-842.

8. Akira M. Computed tomography and pathologic findings in fulminant forms of idiopathic interstitial pneumonia. J Thorac Imaging 1999; 14: 76-84.

9. Harris NL, McNeely W, Shepard JA, Ebeling S, Ellender S, Peters C. Case records of the Massachusetts General Hospital. N Engl J Med 2002; 347: 2149-2157.

10. Panos RJ, Mortenson RL, Niccoli SA, King TE. Clinical deterioration in patients with idiopathic pulmonary fibrosis: causes and assessment. Am J Med 1990; 4: 396-404.

11. Kitaichi M. Pathologic features and classification of interstitial pnueumonia of unknown etiology. Bull Chest Dis Res Inst Kyoto Univ 1990; 23: 1-18.

12. Maynaud C, Cadranel J. A persistent challenge: the diagnosis of respiratory disease in the non-AIDS immunocompromised host. Thorax: 2000: 511-517.

13. Martino R, Ramila E, Rabella N, et al. Respiratory virus infection in adults with hematologic malignancies: a prospective study. Clin Infect Dis 2003; 36: 1-8.

14. Bonaccorsi A, Cancellieri A, Chilosi M, et al. Acute interstial pneumonia: report of a series. Eur Respir J 2003; 21: 15 .

15. Ultz JP, Ryu JH, Douglas WW, et al. High short term mortality following lung biopsy for usual interstitial pneumonia. Eur Respir J 2001; 17: 175-179. 